\title{
Pilot study: rapidly cycling hypobaric pressure improves pain after 5 days in adiposis dolorosa
}

This article was published in the following Dove Press journal:

Journal of Pain Research

19 August 2010

Number of times this article has been viewed

\author{
Karen L Herbst' \\ Thomas Rutledge ${ }^{2}$ \\ 'Department of Medicine, University \\ of California, San Diego, California, \\ USA; ${ }^{2}$ Department of Psychiatry, \\ University of California, San Diego, \\ California, USA
}

\begin{abstract}
Adiposis dolorosa (AD) is a rare disorder of painful nodular subcutaneous fat accompanied by fatigue, difficulty with weight loss, inflammation, increased fluid in adipose tissue (lipedema and lymphedema), and hyperalgesia. Sequential compression relieves lymphedema pain; we therefore hypothesized that whole body cyclic pneumatic hypobaric compression may relieve pain in AD. To avoid exacerbating hyperalgesia, we utilized a touch-free method, which is delivered via a high-performance altitude simulator, the Cyclic Variations in Altitude Conditioning ${ }^{\mathrm{TM}}\left(\mathrm{CVAC}^{\mathrm{TM}}\right)$ process. As a pilot study, 10 participants with $\mathrm{AD}$ completed pain and quality of life questionnaires before and after 20-40 minutes of CVAC process daily for 5 days. Participants lost weight $(195.5 \pm 17.6-193.8 \pm 17.3 \mathrm{lb} ; P=0.03)$, and bioimpedance significantly decreased $(510 \pm 36-490 \pm 38 \mathrm{ohm} ; P=0.01)$. There was a significant decrease in scores on the Pain Catastrophizing Scale $(P=0.039)$, in average $(P=0.002)$, highest $(P=0.029)$, lowest $(P=0.04)$, and current pain severity $(P=0.02)$ on the Visual Analogue Scale, but there was no change in pain quality by the McGill Pain Questionnaire. There were no significant changes in total and physical SF-36 scores, but the mental score improved significantly $(P=0.049)$. There were no changes in the Pain Disability Index or Pittsburgh Sleep Quality Index. These data present a potential, new, noninvasive means of treating pain in AD by whole body pneumatic compression as part of the CVAC process. Although randomized, controlled trials are needed to confirm these data, the CVAC process could potentially help in treating $\mathrm{AD}$ pain and other chronic pain disorders.
\end{abstract}

Keywords: bioimpedance, chronic pain, lipedema

\section{Introduction}

Adiposis dolorosa (AD) is considered a rare disorder by the National Institutes of Health and the National Organization for Rare Disease; in Lund, Sweden, 0.1\% of the population is affected. ${ }^{1}$ The pain associated with $\mathrm{AD}$ is in subcutaneous fat, but patients also complain of fatigue, cognitive changes, sleep disturbance, shortness of breath, rapid heart rate, gastrointestinal complaints, and myalgias and arthralgias. Hyperalgesia is prominent, similar to signs and symptoms associated with fibromyalgia, ${ }^{2}$ but with the difference that the physical examination is greatly abnormal in AD compared with essentially normal in fibromyalgia. The pain in $\mathrm{AD}$ varies in intensity and type, can occur in flares, and is associated with inflammation ${ }^{3}$ and fluid in adipose tissue called lipedema, ${ }^{4}$ which can progress to lymphedema; seroma formation is common after adipose resection. ${ }^{3,4}$

Current treatments for AD include surgical excision or liposuction, ${ }^{5-7}$ intravenous lidocaine ${ }^{8}$ topical analgesics, ${ }^{9,10}$ infliximab and methotrexate, ${ }^{11}$ and pregabalin combined with manual lymphatic drainage therapy. ${ }^{12}$ Intravenous lidocaine is not effective
Correspondence: Karen L Herbst 3350 La Jolla Village $\operatorname{Dr}$ ( I I IG), San Diego, CA 92161, USA

Tel + I 858-552-8585 (x 7384)

Fax + I 858-642-6242

Email karen.herbst@va.gov 
for many patients with $\mathrm{AD}$; topical analgesics provide modest relief; and the immunosuppressive combination of infliximab and methotrexate risks complications, such as infection, and has only shown benefit in a single case to date. External pneumatic compression is known to relieve pain associated with edema. ${ }^{13,14}$ Although whole body external pneumatic compression is available, ${ }^{15}$ the neck and head are not treated raising a concern for increasing fluid in these areas and exacerbating the fatigue, cognitive changes, and sleep disturbances in AD. Manual lymphatic drainage is also effective for pain relief in $\mathrm{AD} ;{ }^{12}$ however, only small areas of the body can be treated at a time. In addition, individuals with $\mathrm{AD}$ may not tolerate external compression because of hyperalgesia.

The Cyclic Variations in Altitude Conditioning ${ }^{\mathrm{TM}}$ (CVAC) (CVAC Systems, Inc; Temecula, California, USA) process is an alternative and potentially revolutionary method of touchfree cyclic hypobaric pneumatic compression for treatment of tissue edema and, therefore, edema-associated pain. During the CVAC process, an individual sits within the CVAC altitude simulator while pressure cycles 300 to 500 times in a session simulating altitudes up to 3,200 m. At higher altitude levels (up to $6,858 \mathrm{~m}$ ), the dynamic change in hypobaric pressure over a larger range of altitudes provides an intermittent hypobaric hypoxic experience. The CVAC process has been shown to improve blood oxygen saturation up to $6 \%$ by pulse oximetry, consistent with a rapid, enduring, and beneficial altitude response. ${ }^{16}$ We hypothesized that the CVAC process would improve pain and quality of life in $\mathrm{AD}$ patients as measured by validated questionnaires.

\section{Materials and methods Participants}

Ten consecutive participants were recruited and completed the study with no dropouts. The average age of the 4 men and 6 women in the study was $48 \pm 3.6$ years ranging from 31 to 72 years (Table 1). All participants were Caucasian and nonHispanic with the exception of 1 male Hispanic. The average weight was $88.7 \pm 8 \mathrm{~kg}$, and the body mass index was $28.3 \pm 1.8 \mathrm{~kg} / \mathrm{m}^{2}$. The majority of subjects took multiple medications and had additional comorbidities. Half of the subjects carried a diagnosis of fibromyalgia.

\section{Study protocol}

$\mathrm{AD}$ is a rare disorder; therefore, the number of affected participants in a given area may be small. This study was limited to individuals with $\mathrm{AD}$ interested in research, in our database within the local area or those requesting participation in research who were from out of town and could travel and stay for the week-long study. This study was approved by the Institutional Review Board at the University of California, San Diego (UCSD) and by the Veterans Affairs San Diego Healthcare System (VASDHS) Research and Development

Table I Demographics, medications, and comorbidities of participants with AD

\begin{tabular}{|c|c|c|c|c|}
\hline Subject no. & Sex & Age & Medications & Comorbidities \\
\hline 1 & M & 46 & $\begin{array}{l}\text { Aspirin; eszopiclone; famotidine; loratidine; } \\
\text { metoprolol; omeprazole; tramadol; valsartan }\end{array}$ & $\begin{array}{l}\text { Esophageal candidiasis; gastritis; HTN; lipoma } \\
\text { resection }\end{array}$ \\
\hline 2 & $\mathrm{~F}$ & 56 & $\begin{array}{l}\text { Alprazolam; cephalexin; cyclobenzaprine; diazepam; } \\
\text { duloxetine; hydroxyzine; modafinil; opioid; narcotic; } \\
\text { pantoprazole; zolpidem }\end{array}$ & $\begin{array}{l}\text { Achilles tendon repair; familial multiple lipomatosis; } \\
\text { lipedema; lipolymphedema; morbid obesity; } \\
\text { total knee replacement }\end{array}$ \\
\hline 3 & M & 37 & Medical marijuana; opioid; narcotic & Fibromyalgia \\
\hline 4 & $\mathrm{~F}$ & 36 & None & $\begin{array}{l}\text { Fibromyalgia; hysterectomy; lipoma resection; } \\
\text { pseudotumor cerebri }\end{array}$ \\
\hline 5 & $\mathrm{~F}$ & 39 & Zolpidem & $\begin{array}{l}\text { Adipose liposuction; endometriosis; IBS; insomnia; } \\
\text { lipoma resection; Raynaud's disease }\end{array}$ \\
\hline 6 & $\mathrm{~F}$ & 59 & $\begin{array}{l}\text { Aripiprazole; lidocaine gel; lovastatin; omeprazole; } \\
\text { opioid; narcotic; pregabalin }\end{array}$ & $\begin{array}{l}\text { Depression; fibromyalgia; GERD; dyslipidemia; bilateral } \\
\text { TMJ surgery }\end{array}$ \\
\hline 7 & M & 51 & $\begin{array}{l}\text { Carbamazepine; gabapentin; metformin; } \\
\text { opioid narcotic; temazepam; testosterone; } \\
\text { venlafaxine; warfarin; zonisamide }\end{array}$ & $\begin{array}{l}\text { Cognitive dysfunction; depression; familial } \\
\text { multiple lipomatosis; fibromyalgia; hypogonadism; } \\
\text { hypovitaminosis D; insomnia }\end{array}$ \\
\hline 8 & M & 47 & Cetirizine; lorazepam; omeprazole & Environmental allergies; familial multiple lipomatosis \\
\hline 9 & $\mathrm{~F}$ & 72 & $\begin{array}{l}\text { Allopurinol; amlodipine; aspirin; } \\
\text { colchicine; lorazepam; metoprolol; sucralfate; }\end{array}$ & $\begin{array}{l}\text { Chronic back pain; Dupuytren's contracture; } \\
\text { dyslipidemia; HTN; osteoarthritis }\end{array}$ \\
\hline
\end{tabular}


Committee. All participants provided written and informed consent prior to participation. Subject visits were at the Center for Metabolic Research as part of the Special Diagnostic and Treatment Unit at the VASDHS. Participants were enrolled if they had a diagnosis of AD (confirmed by 1 of the authors, $\mathrm{KLH})$. Anecdotal data suggested that more than one 20-minute CVAC process exposure beyond the acclimation phase (day 1) was required for a change in pain; therefore, we chose 5 days of CVAC process exposures for this protocol (CVAC Systems, Inc, pers comm). After consent, participants were weighed, and completed questionnaires then underwent the first CVAC process (total of 25 minutes). On days 2-5, participants were exposed to the CVAC process during two 20-minute sessions. On day 5, participants repeated questionnaires and received the final two 20-minute CVAC process exposures.

\section{CVAC process sessions}

The dynamic whole body cyclic pneumatic compression was administered using a high-performance altitude simulator. Participants sit in the simulator that has a large window resembling the canopy windshield of a fighter jet. The CVAC process exposes individual users to various simulated altitudes arranged into precise patterns. Each session consists of between 300 and 500 cyclic altitude changes in a 20-minute period with an average rate of change of $30.5 \mathrm{~m} / \mathrm{s}$ and an approximate cumulative change of $365,760 \mathrm{~m}$ in a 20 -minute session. A proprietary robotic system controls pressure changes within the simulator, whereas the open system prevents carbon dioxide build-up. The dynamic changes result in a pulsatile effect of pressure; at altitudes above 3,200 m (not in this study protocol), there is also a pulsatile effect on oxygen saturation in the blood. ${ }^{16}$ Examples of these dynamic changes have been published previously. ${ }^{16}$ Participants were instructed how to equalize pressures in their ears using the jaw jack, the Valsalva maneuver, and side-to-side neck extension as the Eustachian tubes change shape with changing pressures. For this study, on day 1, participants were initially exposed to the CVAC process in 5 stages (Tier 1 ; \#1-5) of 5 minutes' duration each. The maximal altitude for this Tier 1 stage is 3,200 m. On each subsequent day, participants were given a maximum of two 20-minute sessions on Tier 2, with a maximum altitude also of 3,200 $\mathrm{m}$.

\section{Bioelectrical impedance analysis}

Bioelectrical impedance analysis (BIA) measurements were taken by using the Tanita ${ }^{\circledR}$ leg-to-leg system (model TBF-300A; Arlington Heights, Illinois, USA). The Tanita analyzer measures lower-body resistance between the right and the left legs as the individual stands on the electrode plates of the analyzer. During the 5-day protocol, participants were measured at the same time each morning after emptying the bladder, and while standing erect, in bare feet, on the analyzer's footpads wearing street clothes or a gown. The system's 2 electrodes are in the form of stainless steel footpads. Leg-to-leg impedance and body mass are simultaneously measured as the subject's bare feet make pressure contact with the electrodes and digital scale. The body fat monitor or analyzer automatically measures weight and then impedance. Computer software (a microprocessor) imbedded in the product uses the measured impedance, the subject's gender, height, fitness level and age, which have been preprogrammed, and the weight to determine body fat percentage based on equation formulas. Through multiple regression analysis, Tanita has derived standard formulas to determine body fat percentage. Tanita's equations are generalized for standard adults and athletes. The technology determines the electrical impedance of body tissues, which provides an estimate of total body water (TBW). Using these values of TBW derived from BIA, fat-free mass (FFM) and body fat may then be estimated. The standard error of estimate or prediction error for BIA is about $3.5 \% .{ }^{17}$

\section{Questionnaires}

On days 1 and 5 of the study, participants completed measures of pain severity (McGill Pain Questionnaire), ${ }^{18}$ pain-related symptoms (Pain Catastrophizing Scale $[\mathrm{PCS}]^{19}$ and Pain Disability Index [PDI]), and quality of life (the Pittsburgh Sleep Quality Index [PSQI] ${ }^{20}$ and SF-36). Participants completed the Visual Analogue Scale (VAS) as a daily measure of pain severity. We selected the above questionnaires based on the criteria of being validated instruments, widely used in studies of chronic pain patients, that captured the spectrum of pain intensity, and the functional aspects of pain believed to potentially improve as a result of the CVAC process exposures.

The McGill Pain Questionnaire is a widely used measure assessing the quality and intensity of pain. The VAS is a single-item measure wherein respondents rate their pain intensity on a $0-10$ scale, with 0 indicating no pain and 10 representing the worst pain imaginable. The PCS is a 13-item questionnaire assessing pain perceptions in the areas of rumination, magnification, and helplessness. Higher scores indicate a greater tendency to catastrophize pain symptoms. The PDI is a 7-item instrument that quantifies the impact of pain on the respondent in areas of living including family, recreation, social, occupational, sexual, self-care, and life support activities. The PSQI was developed to measure 
sleep quality during the previous month covering domains including subjective sleep quality, sleep latency, sleep duration, habitual sleep efficiency, sleep disturbances, use of sleep medications, and daytime dysfunction. Finally, the SF-36 is a multidimensional health survey yielding an overall functional health score and separate physical and mental health dimension scores.

\section{Data analyses}

Data are presented as average \pm standard error of the mean. Statistical analyses consisted of repeated measures analysis of variance (ANOVA) models to compare mean changes between day 1 and day 5 on the measured pain, psychosocial, and metabolic indices. For the VAS data, wherein pain scores were available for each of the 5 participation days, we used repeated measures ANOVA to assess trends in average, high, and low pain scores across the length of the study protocol. In each of the 3 VAS repeated measures models, we assessed the assumption of sphericity using Mauchly's test. This assumption was met in all cases. These analyses were performed using GraphPad Prism version 5.00 (GraphPad Software; San Diego, California, USA) for Windows. For the purpose of some of the graphical illustrations, we depicted results in units of percent changes, calculated as the absolute mean difference in day 1 vs day 5 divided by the day 1 mean ([D1-D5]/D1). A $P$ value of 0.05 was used as the criterion for declaring statistical significance.

\section{Results \\ CVAC process}

The average number of CVAC sessions completed during days $1-5$ was $9.4 \pm 0.5$. One participant was able to complete only 1 session daily due to difficulty in equilibrating ear pressure. Another completed only 8 sessions for the same reason. No side effects occurred.

\section{Questionnaires}

A comparison of pre-CVAC vs post-CVAC process exposures McGill Pain Questionnaire scores indicated a significant decrease in participant's current pain severity $(3.1 \pm 0.3$ to $2.0 \pm 0.2$ from a total of $5 ; P=0.02$ ), but no change in ratings of pain quality or in worst and least pain reports (Figure 1). Scores on the PCS also significantly decreased on day 5 compared with day $1(28.2 \pm 3.5$ to $25.2 \pm 2.9 ; P=0.039)$. Undergoing the CVAC process also resulted in significantly reduced average ( $5.6 \pm 0.6$ to $4.2 \pm 0.6 ; P=0.002)$, highest ( $7 \pm 0.7$ to $5.7 \pm 0.7 ; P=0.029)$, and lowest pain $(4.4 \pm 0.5$ to $3.4 \pm 0.4 ; P=0.043$ ) levels on day 5 compared with day 1 .
A trend analysis for the daily average VAS data indicated significant linear (overall decrease across the 5 days; $P=0.002$ ) and quadratic (flatter means in first days of pain reports followed by larger decreases later; $P=0.013$ ) patterns of changes over 5 days (Figure 1). There was a significant linear $(P=0.043)$ but not quadratic decrease for highest pain by the VAS over 5 days, and again a significant linear $(P=0.028)$ and quadratic $(P=0.03)$ pattern of decreases were evident in lowest experienced pain over 5 days on the VAS. There was no change in the overall SF-36 score or physical functioning, but mental functioning increased significantly (32.8 \pm 4.8 to $38.8 \pm 4.7 ; P=0.049)$. There were no significant changes in the PDI or PSQI on day 5 compared with day 1 (data not reported). Data from the study questionnaires are illustrated in Figure 1.

\section{Weight and BIA}

Subject weight significantly decreased from day 1 to day 5 ( $88.7 \pm 8$ to $87.8 \pm 7.8 \mathrm{~kg} ; P=0.03$; Figure 1$)$. Although there was a nonsignificant increase in percent fat $(32.4 \pm 3.4 \%$ to $35.4 \pm 3.7 \%)$ and total fat mass $(27.2 \pm 3.9$ to $32.9 \pm 6.2 \mathrm{~kg})$, there was a trend to decrease FFM $(55 \pm 3.8$ vs $54.9 \pm 3.3 \mathrm{~kg}$; $P=0.053)$ by BIA. Bioimpedance decreased significantly from $510 \pm 36$ to $490 \pm 38 \mathrm{ohm}(P=0.014$; Figure 1$)$ with no significant change in calculated TBW $(40.8 \pm 2.6$ to $41.4 \pm 2.6 \mathrm{~kg})$.

\section{Discussion}

We demonstrated that changing air pressure around participants with AD from ambient altitude (about $60 \mathrm{~m}$ above sea level in San Diego, California, USA) to 3,200 m, 300-500 times in a 20-minute session, for up to 2 sessions daily (total of 40 minutes), over 5 days significantly reduced pain perceptions and intensity. The average altitude for the sessions over the 5 -day period was approximately $1,828 \mathrm{~m}$. This pneumatic compression benefit on pain reports manifested across several distinct measures including VAS-measured pain intensity, lower current pain levels as captured by the McGill Pain Questionnaire, reduced tendencies to catastrophize pain symptoms on the PCS, and greater quality of life as indicated by improved mental health function scores on the SF-36. Given that both the pain patterns on the average and the low VAS scores had significant linear and quadratic changes, our data suggest that pain changes really did not manifest until day 3 or 4 . These data could infer that a 1-day CVAC process intervention would not be sufficient to alter pain status. On the contrary, we saw no evidence of pain scores leveling off during days 4-5, so an even longer CVAC protocol might 
A

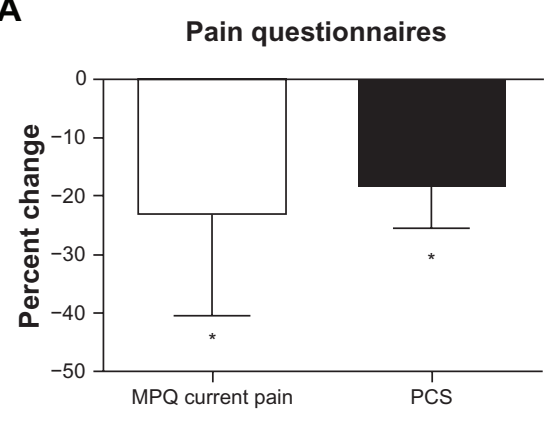

C

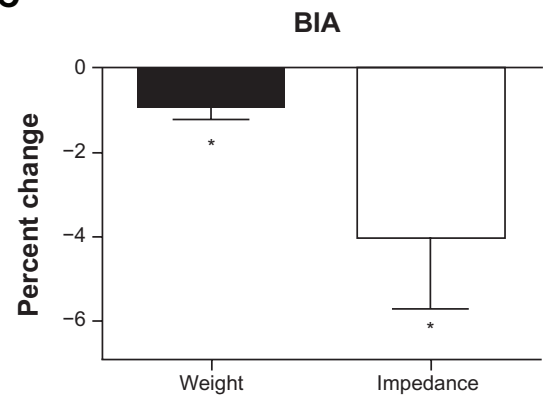

B

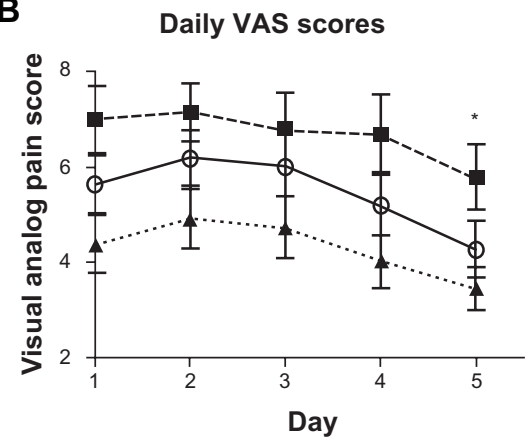

D

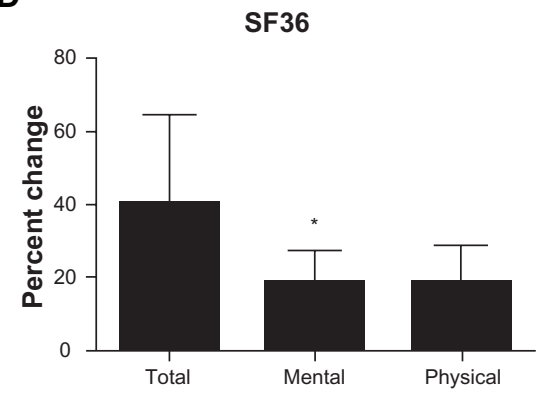

Figure I Change in pain questionnaire scores, weight, and bioimpedance before and after CVAC process exposures. A, MPQ (change in current pain) and PCS. B, Mean daily scores for average (open circles, unbroken line), highest (filled squares, dashed line), and lowest pain (filled triangle, dotted line) by the VAS. C, Change in percent weight and impedance from bioimpedance analysis. D, Percent change in total, mental, and physical SF-36 values.

Abbreviations: CVAC, Cyclic Variations in Altitude Conditioning; MPQ, McGill pain questionnaire; PCS, pain catastrophizing Scale; VAS, visual analog scale; ANOVA, analysis of variance; BIA, bioelectrical impedance analysis.

* $P<0.05$ comparing values from day I to day 5 by repeated measures ANOVA; actual $P$-values available in the text.

yield additional benefits. Although these results are limited by the small sample, short-term treatment, and nonrandomized design of this pilot study, the observation that widespread pain-related benefits can be achieved in a brief CVAC process intervention is supportive of further research attention to this novel treatment for AD.

At least 3 potential mechanisms may account for the observed improvements in pain reports in this study. First, tissue edema likely decreased, as supported by a significant decrease in weight in participants and a significant decrease in impedance; a decrease in impedance indicates less water in tissue. The multiple changes in external pressure during the CVAC process exposure may cause the lymph fluid to move out of the tissue similar to external compression; ${ }^{21}$ this pneumatic displacement of fluid improves pain in lymphedema. ${ }^{22}$ Another possible mechanism to explain improved pain in participants with $\mathrm{AD}$ could be through improved blood flow by the intermittent pneumatic compression, ${ }^{23}$ consistent with the CVAC process; this could allow for a secondary decrease in tissue edema. The CVAC process has also been demonstrated to increase arterial oxygen saturation. ${ }^{16}$ Muscle contraction improving lymph flow and oxygenation also occurs with exercise. ${ }^{24}$ Pain in lipedema is thought to result from hypoxia, inflammation, and necrosis of adipocytes; ${ }^{25}$ therefore, improved blood flow and oxygen saturation may decrease hypoxia in the painful adipose tissue. ${ }^{26}$ If these mechanistic theories are confirmed, the CVAC process may also hold treatment potential for other chronic pain conditions in which impaired blood flow and tissue edema are contributing factors. For example, improved blood flow may be a mechanism through which exercise improves pain and quality of life among fibromyalgia patients. ${ }^{27}$ To date, however, the CVAC process has not been tested in fibromyalgia populations, although 5 of 10 participants in this study had a diagnosis of fibromyalgia. Future work could focus on pain relief after CVAC process exposure compared with exercise or combined with exercise. Pain pathways could also be assessed systematically as they relate to pneumatic compression or exercise.

In contrast to our observations of consistently favorable changes in pain reports following the 5-day CVAC process intervention, there was no evidence to suggest improvements in areas of pain disability, sleep, or physical function from this protocol. Although it is possible that the lack of improvement in these areas represents limitations in CVAC process treatment, an alternative interpretation is that the absence of changes in the latter dimensions was a result of the brevity of the intervention. A longer measurement protocol may 
be required in order to assess the potential for meaningful change in the areas of sleep quality and quantity, family, social and sexual relationships, occupational functioning, and self-care. Additional research will be required to compare the merits of these interpretive hypotheses.

We also observed some preliminary evidence for CVAC process-induced body weight changes as measured by bioimpedance technology. Weight significantly decreased for the participants in this pilot study over 5 days. Interestingly, although weight significantly decreased after CVAC process exposures, fat mass and fat percent increased and FFM significantly decreased as measured by BIA. Bioimpedance analysis is a widely used method to estimate body composition. When a current is passed through the body, the water-containing tissues primarily conduct the electrical current so that impedance is higher with increased FFM, which is $73 \%$ hydrated; ${ }^{28,29}$ impedance is also increased in lymphedema. ${ }^{30}$ In other words, impedance increases with increased water in tissues. In BIA, higher TBW results in a measure of lower body fat percentage; less water increases the percentage of body fat and decreases calculated FFM. There is currently no algorithm for BIA that provides a volume in liters. ${ }^{29}$ In our participants, although weight significantly decreased after CVAC process exposures, fat mass and fat percent increased and FFM decreased consistent with a significant decrease in impedance. These data suggest that the CVAC process decreases tissue fluid in $\mathrm{AD}$ accounting for a significant decrease in weight loss. The CVAC process of gentle pneumatic compression cycled 300-500 times in a 20-minute period repeated on an average of 9 times in 1 week, therefore, decreased tissue fluid weight in our participants with $\mathrm{AD}$ while decreasing pain. These changes in BIA measures would best be confirmed by the use of dual X-ray absorptiometry for body composition, perometry for limb volume measurements, and deuterium isotope dilution as a measure of TBW before and after the CVAC process.

In conclusion, the CVAC process, which is touchless, cycled, pneumatic, hypobaric compressions administered via a high-performance altitude simulator, may decrease tissue fluid and improve oxygen saturation resulting in decreased pain in people with AD. Although randomized, controlled trials are needed to confirm these data, the CVAC process could potentially help in treating $\mathrm{AD}$, related chronic pain disorders, and painful lipedema.

\section{Acknowledgments}

The authors thank Phil Peck, Torri Middlebrooks, and Amanda Wirtz for conducting CVAC sessions; the Center for Metabolic Research in the VASDHS for the use of their facilities; and CVAC Systems, Inc, for the use of the CVAC altitude simulator. The UCSD General Clinical Research Center and the NIDDK supported KLH by Public Health Grant 5M01 RR000827and grant K23 DK 065038-05, respectively.

\section{Disclosure}

The authors report no conflicts of interest in this work.

\section{References}

1. Brorson H, Fagher B. Dercum's disease. Fatty tissue rheumatism caused by immune defense reaction? Lakartidningen. 1996;93(15):1430, 1433-1436.

2. Stormorken H, Brosstad FHS. The fibromyalgia syndrome: A member of the painful lipomatosis family? In: Pederson JA, editor. New Research on Fibromyalgia. New York: Nova Science Publishers; Inc; 2006.

3. Herbst KL, Asare-Bediako S. Adiposis Dolorosa is more than painful fat. Endocrinologist. 2007;17(6):326-344.

4. Dercum FX. A subcutaneous connective-tissue dystrophy of the arms and back, associated with symptoms resembling myxoedema. Univ Med Mag Philadelphia. 1888;1:140-150.

5. Berntorp E, Berntorp K, Brorson H, Frick K. Liposuction in Dercum's disease: impact on haemostatic factors associated with cardiovascular disease and insulin sensitivity. J Intern Med. 1998;243(3):197-201.

6. Brorson H, Aberg M, Fagher B. Liposuction in adiposis dolorosa (morbus Dercum) - an effective therapy. Ugeskr Laeger. 1992;154(27): 1914-1915.

7. DeFranzo AJ, Hall JH Jr, Herring SM. Adiposis dolorosa (Dercum's disease): liposuction as an effective form of treatment. Plast Reconstr Surg. 1990;85(2):289-292.

8. Petersen P, Kastrup J. Dercum's disease (adiposis dolorosa). Treatment of the severe pain with intravenous lidocaine. Pain. 1987;28(1):77-80.

9. Desai MJ, Siriki R, Wang D. Treatment of pain in Dercum's disease with Lidoderm (lidocaine 5\% patch): a case report. Pain Med. 2008;9(8):1224-1226. Epub 2008 Mar 11.

10. Reggiani M, Errani A, Staffa M, Schianchi S. Is EMLA effective in Dercum's disease? Acta Derm Venereol. 1996;76(2):170-171.

11. Singal A, Janiga J, Bossenbroek N, Lim H. Dercum's disease (adiposis dolorosa): a report of improvement with infliximab and methotrexate. J Eur Acad Dermatol Venereol. 2007;21(5):717.

12. Lange U, Oelzner P, Uhlemann C. Dercum's disease (Lipomatosis dolorosa): successful therapy with pregabalin and manual lymphatic drainage and a current overview. Rheumatol Int. 2008;29(1):17-22. Epub 2008 Jul 5.

13. Hamner JB, Fleming MD. Lymphedema therapy reduces the volume of edema and pain in patients with breast cancer. Ann Surg Oncol. 2007;14(6):1904-1908. Epub 2007 Mar 8.

14. Tamir L, Hendel D, Neyman C, Eshkenazi AU, Ben-Zvi Y, Zomer R. Sequential foot compression reduces lower limb swelling and pain after total knee arthroplasty. J Arthroplasty. 1999;14(3): 333-338.

15. Morris RJ. Intermittent pneumatic compression - systems and applications. J Med Eng Technol. 2008;32(3):179-188.

16. Hetzler RK, Stickley CD, Kimura IF, et al. The effect of dynamic intermittent hypoxic conditioning on arterial oxygen saturation. Wilderness Environ Med. 2009;20(1):26-32.

17. Heymsfield SB, Wang Z, Visser M, Gallagher D, Pierson RN Jr. Techniques used in the measurement of body composition: an overview with emphasis on bioelectrical impedance analysis. Am J Clin Nutr. 1996;64 Supp1 3:S478-S484.

18. Melzack R. The McGill pain questionnaire: from description to measurement. Anesthesiology. 2005;103(1):199-202.

19. Sullivan MJL, Bishop S, Pivik J. The pain catastrophizing scale: development and validation. Eur J Psychol Assess. 1995;7:524-532. 
20. Buysse DJ, Reynolds CF III, Monk TH, Berman SR, Kupfer DJ. The Pittsburgh Sleep Quality Index: a new instrument for psychiatric practice and research. Psychiatry Res. 1989;28(2):193-213.

21. McGeown JG, McHale NG, Thornbury KD. The role of externa compression and movement in lymph propulsion in the sheep hind limb. J Physiol. 1987;387:83-93.

22. Yamazaki Z, Idezuki Y, Nemoto T, Togawa T. Clinical experiences using pneumatic massage therapy for edematous limbs over the last 10 years. Angiology. 1988;39(2):154-163.

23. Delis KT, Labropoulos N, Nicolaides AN, Glenville B, Stansby G. Effect of intermittent pneumatic foot compression on popliteal artery haemodynamics. Eur J Vasc Endovasc Surg. 2000;19(3):270-277.

24. Havas E, Parviainen T, Vuorela J, Toivanen J, Nikula T, Vihko V. Lymph flow dynamics in exercising human skeletal muscle as detected by scintography. J Physiol. 1997;504(Pt 1):233-239.
25. Fife CE, Maus EA, Carter MJ. Lipedema: a frequently misdiagnosed and misunderstood fatty deposition syndrome. Adv Skin Wound Care. 2010;23(2):81-92; quiz 93-84.

26. Ye J. Emerging role of adipose tissue hypoxia in obesity and insulin resistance. Int J Obes (Lond). 2009;33(1):54-66.

27. Fontaine KR, Conn L, Clauw DJ. Effects of lifestyle physical activity on perceived symptoms and physical function in adults with fibromyalgia: results of a randomized trial. Arthritis Res Ther. 2010;12(2):R55.

28. Cornish B. Bioimpedance analysis: scientific background. Lymphat Res Biol. 2006;4(1):47-50.

29. Earthman C, Traughber D, Dobratz J, Howell W. Bioimpedance spectroscopy for clinical assessment of fluid distribution and body cell mass. Nutr Clin Pract. 2007;22(4):389-405.

30. Warren AG, Janz BA, Slavin SA, Borud LJ. The use of bioimpedance analysis to evaluate lymphedema. Ann Plast Surg. 2007;58(5):541-543.
Journal of Pain Research

\section{Publish your work in this journal}

The Journal of Pain Research is an international, peer-reviewed, open access, online journal that welcomes laboratory and clinical findings in the fields of pain research and the prevention and management of pain. Original research, reviews, symposium reports, hypothesis formation and commentaries are all considered for publication.

\section{Dovepress}

The manuscript management system is completely online and includes a very quick and fair peer-review system, which is all easy to use. Visit http://www.dovepress.com/testimonials.php to read real quotes from published authors.

\footnotetext{
Submit your manuscript here: http://www.dovepress.com/journal-of-pain-research-journal
} 